PROCEEDINGS OF THE

AMERICAN MATHEMATICAL SOCIETY

Volume 131, Number 12, Pages 3689-3692

S 0002-9939(03)07047-3

Article electronically published on April 30, 2003

\title{
A PROPERTY OF WEAKLY KRULL DOMAINS
}

\author{
D. D. ANDERSON AND MUHAMMAD ZAFRULLAH
}

(Communicated by Bernd Ulrich)

\begin{abstract}
We show that a weakly Krull domain $D$ satisfies $(*)$ : for every pair $a, b \in D \backslash\{0\}$ there is an $n=n(a, b) \in \mathbb{N}$ such that $\left(a, b^{n}\right)$ is $t$-invertible. For $D$ Noetherian, $D$ satisfies $(*)$ if and only if every grade-one prime ideal of $D$ is of height one. We also show that a modification of $(*)$ can be used to characterize Noetherian domains that are one-dimensional.
\end{abstract}

Let $D$ be an integral domain with quotient field $K$, and let $X^{1}(D)$ denote the set of height-one prime ideals of $D$. The domain $D$ is said to be a weakly Krull domain if $D$ is a locally finite intersection of its localizations at members of $X^{1}(D)$, i.e., if $D=\bigcap_{P \in X^{1}(D)} D_{P}$ and this intersection is locally finite. Indeed, a weakly Krull domain $D$ is the well-known Krull domain if we insist that $D_{P}$ is a discrete rank-one valuation domain for each $P \in X^{1}(D)$. And if we settle for a weakly Krull domain $D$ such that $D_{P}$ is a valuation domain for each $P \in X^{1}(D)$, we get a generalized Krull domain of Ribenboim [R]. Obviously, a Noetherian domain is a weakly Krull domain if each grade-one prime ideal of $D$ is of height one. The converse is also true. Weakly Krull domains have recently been of interest in connection with the study of factorization properties of algebraic order, see, e.g., Picavet-L'Hermitte [P]. The purpose of this short note is to bring to light the following interesting property of weakly Krull domains. Let $D$ be a weakly Krull domain; then for every pair $a, b \in$ $D^{*}=D \backslash\{0\}$, there is a natural number $n=n(a, b)$ such that $\left(a, b^{n}\right)$ is a $t$-invertible ideal of $D$. What may be of general interest here is that (within the framework of Noetherian domains) Noetherian weakly Krull domains are actually characterized by this somewhat weak property and so are one-dimensional Noetherian domains on replacing " $t$-invertible" by "invertible".

To keep the note short, we shall assume a working knowledge of star-operations as provided by Gilmer's book [G, Sections 32 and 34] and of the notion of $t$-invertibility as in [Z]. However, we recall for the reader's convenience the following facts. Let $F(D)$ denote the set of nonzero fractional ideals. Then for $I \in F(D), I^{-1}=D:_{K} I$ and the function $I \mapsto I_{v}=\left(I^{-1}\right)^{-1}$ on $F(D)$ is a star-operation called the $v$ operation. The operation on $F(D)$ defined by $A \mapsto A_{t}=\bigcup\left\{\left(a_{1}, a_{2}, \ldots, a_{n}\right)_{v} \mid a_{i} \in\right.$ $I \backslash\{0\}, n \in \mathbb{N}\}$ is called the $t$-operation. A fractional ideal $I$ is a $v$-ideal ( $t$-ideal) if $I_{v}=I$ (resp., $I_{t}=I$ ) and an ideal $M$ maximal among integral $t$-ideals is a prime ideal called a maximal $t$-ideal. A fractional ideal $I$ is $t$-invertible if $\left(I I^{-1}\right)_{t}=D$. Finally, we add the following: (1) every minimal prime of a principal ideal is a

Received by the editors August 12, 2002.

2000 Mathematics Subject Classification. Primary 13F05.

Key words and phrases. Weakly Krull. 
prime $t$-ideal $[\mathrm{HH}],(2)$ if $D$ is a weakly Krull domain, then $X^{1}(D)$ is precisely the set of maximal $t$-ideals of $D$ [AMZ, Theorems 3.1 and 4.3], and (3) a finitely generated ideal $A$ of a domain $D$ is $t$-invertible if and only if $A D_{P}$ is principal for each maximal $t$-ideal $P$ of $D$ [MMZ, Lemma 1.5].

We start by proving the result mentioned above.

Theorem 1. Let $D$ be a weakly Krull domain, and let $a, b$ be two nonzero elements of $D$. Then there exists a natural number $n=n(a, b)$ such that $\left(a, b^{n}\right)$ is $t$-invertible.

Proof. If either of $a, b$ is a unit, then $\left(a, b^{m}\right)=D$ for every natural number $m$ and hence is $t$-invertible. So let us concentrate on the case when both $a$ and $b$ are nonunits. If $P$ is a height-one prime ideal of $D$ such that $(a, b) \nsubseteq P$, then too $(a, b) D_{P}=D_{P}=\left(a, b^{m}\right) D_{P}$ for all natural numbers $m$. Now, as we are dealing with a weakly Krull domain, there can be only a finite number of maximal $t$-ideals (i.e., height-one primes) containing $(a, b)$. Let $P_{1}, P_{2}, \ldots, P_{r}$ be all the height-one primes containing $(a, b)$. Since the $D_{P_{i}}$ are one-dimensional quasi-local (for $i=1,2, \ldots, r$ ), there exist natural numbers $n_{i}$ such that $a \mid b^{n_{i}}$ in $D_{P_{i}}$. Let $n=\max \left\{n_{i}\right\}$. Then for $i=1,2, \ldots, r,\left(a, b^{n}\right) D_{P_{i}}$ is principal. Now having exhausted all the cases we have established that $\left(a, b^{n}\right) D_{P}$ is principal for all height-one primes and hence for all maximal $t$-ideals of $D$. But this means that $\left(a, b^{n}\right)$ is $t$-invertible.

To see that the converse of Theorem 1 is not in general true, let us recall that a domain $D$ is a Prüfer domain if every two-generated nonzero ideal of $D$ is invertible and hence is $t$-invertible. Let $R=\mathbb{Z}+X \mathbb{Q}[X]$, where $\mathbb{Z}$ and $\mathbb{Q}$ are, respectively, the integers and rational numbers. Now this domain is a Bezout domain, i.e., every two-generated ideal is principal [CMZ $]$ and hence is a Prüfer domain. So $R$ satisfies the conclusion of Theorem 1 but $R$ is rather far away from being a locally finite intersection of localizations at height-one primes in that $R$ is not completely integrally closed and hence cannot be expressed as an intersection of localizations at height-one primes. ( $R$ is not completely integrally closed because in $R, 3$ is a nonunit and $3^{n} \mid X$ for all natural $n$.)

Now we present the promised converse of Theorem 1 for Noetherian domains in a somewhat more general setting. Recall that an integral domain $D$ is a Mori domain if $D$ satisfies ACC on integral divisorial ideals. It is well known that a Mori domain $D$ is a locally finite intersection of localizations at its maximal $v$-ideals which happen to be maximal $t$-ideals. Obviously, a Mori domain satisfies ACC on principal ideals and it is well known that every ring of fractions of a Mori domain is again a Mori domain. We say that $D$ is of $t$-dimension one (notation $t$-dim $(D)=1$ ) if every maximal $t$-ideal of $D$ is of height one. Finally, it goes without saying that a Noetherian domain is a Mori domain, although there do exist examples of Mori domains that are not Noetherian. For instance, every Krull domain is Mori. A reader in need of further information on Mori domains may consult Barucci [B].

Proposition 2. Let $D$ be a Mori domain such that for each pair of nonzero nonunits $a, b \in D$, there is $a k=k(a, b)$ such that $\left(a, b^{k}\right)$ is $t$-invertible. Then $t$-dim $(D)=1$.

Proof. Let $P$ be a maximal $t$-ideal of $D$. Suppose that $\mathfrak{h t} P>1$; so there is a nonzero prime ideal $Q \subsetneq P$. Choose $b \in P_{P}-Q_{P}$ and $0 \neq a \in Q_{P}$. Now as $D_{P}$ is a Mori domain and hence satisfies ACC on principal ideals, we cannot have $b^{k} \mid a$ for all natural numbers $k$. So there is a number $h$ such that $b^{h} \nmid a$ in $D_{P}$. Now by the 
condition, $\left(a,\left(b^{h}\right)^{k}\right)$ is $t$-invertible for some $k$ and hence $\left(a,\left(b^{h}\right)^{k}\right) D_{P}$ is principal. Thus $a \mid b^{h k}$. Hence $b^{h k} \in Q_{P}$ and so $b \in Q_{P}$, a contradiction. This forces $P$ to be a height-one prime. Thus every maximal $t$-ideal of $D$ is height-one prime.

Combining Proposition [2 and Theorem [1] we can state the following corollary.

Corollary 3. A Mori domain $D$ has $t$-dimension one if and only if for each pair of nonzero nonunits $a, b \in D$, there exists a natural number $k=k(a, b)$ such that $\left(a, b^{k}\right)$ is t-invertible.

Now the case of Noetherian domains is not too difficult. The maximal primes of principal ideals of a Noetherian domain are indeed maximal divisorial and hence of grade one. So just for the record, we put down the following corollary.

Corollary 4. In a Noetherian domain D, every grade-one prime ideal is of height one (i.e., $D$ is weakly Krull) if and only if for every pair $a, b$ of nonzero nonunits of $D$, there is a natural number $n=n(a, b)$ such that $\left(a, b^{n}\right)$ is $t$-invertible.

We now state a general result for which we can provide a proof without any reference to star-operations. We include the proof here for the readers who may not have time to look into the star-operations.

Theorem 5. A Noetherian domain $D$ is one-dimensional if and only if for each pair of nonzero elements $a, b \in D$, there is a natural number $n=n(a, b)$ such that $\left(a, b^{n}\right)$ is invertible.

Proof. Suppose that $D$ is one-dimensional Noetherian. If either of $a, b$ is a unit, then $\left(a, b^{m}\right)=D$ for every natural number $m$ and hence is invertible. So let us concentrate on the case when both $a$ and $b$ are nonunits. Next, if $P$ is a maximal ideal of $D$ such that $(a, b) \nsubseteq P$, then too $(a, b) D_{P}=D_{P}=\left(a, b^{m}\right) D_{P}$ for all natural numbers $m$. Now as we are dealing with a one-dimensional Noetherian domain, there can be only a finite number of maximal ideals containing $(a, b)$. Let $P_{1}, P_{2}, \ldots, P_{r}$ be all the maximal ideals containing $(a, b)$. Since the $D_{P_{i}}$ are onedimensional quasi-local (for $i=1,2, \ldots, r$ ), there exist natural numbers $n_{i}$ such that $a \mid b^{n_{i}}$ in $D_{P_{i}}$. Let $n=\max \left\{n_{i}\right\}$; then for $i=1,2, \ldots, r,\left(a, b^{n}\right) D_{P_{i}}$ is principal. Now having exhausted all the cases we have established that $\left(a, b^{n}\right) D_{P}$ is principal for all maximal ideals of $D$. But this means that $\left(a, b^{n}\right)$ is invertible.

Conversely, suppose that $D$ is Noetherian and that for all nonzero $a, b \in D$, there is a natural number $n=n(a, b)$ such that $\left(a, b^{n}\right)$ is invertible. Let $P$ be a maximal ideal of $D$. Suppose that $\mathfrak{h t} P>1$; so there is a nonzero prime $Q \subsetneq P$. Choose $b \in P_{P}-Q_{P}$ and $0 \neq a \in Q_{P}$. Now as $D_{P}$ is Noetherian, we cannot have $b^{k} \mid a$ for all natural numbers $k$. So there is a number $h$ such that $b^{h} \nmid a$ in $D_{P}$. Now by the condition, $\left(a,\left(b^{h}\right)^{k}\right)$ is invertible for some $k$ and hence $\left(a, b^{h k}\right)$ is principal. Thus $a \mid b^{h k}$. Hence $b^{h k} \in Q_{P}$ and so $b \in Q_{P}$, a contradiction.

Indeed, using arguments similar to the ones given in the above theorem, we can show that a Mori (weakly Krull) domain $D$ is one-dimensional if and only if for each pair $a, b \in D \backslash\{0\}$, there is a natural number $n=n(a, b)$ such that $\left(a, b^{n}\right)$ is invertible. (For the case of a weakly Krull domain $D$, note that if $a$ is a nonunit of $D$, then $a$ is contained in a minimal prime of $D$ and hence $\bigcap_{n=1}^{\infty}\left(a^{n}\right)=0$ Q Corollary 1.4].) For the reader wanting a version of Theorem 5 allowing zero divisors, we offer the following result. 
Theorem 6. For a Noetherian ring $R$ the following are equivalent:

(1) For $a, b \in R$, there exists a natural number $n=n(a, b)$ so that $\left(a, b^{n}\right)$ is locally principal.

(2) $R$ is a finite direct product of zero-dimensional local rings and one-dimensional integral domains.

Proof. (1) $\Rightarrow(2)$ Let $M$ be a maximal ideal of $R$. Then for $a, b \in R_{M}$, there exists an $n=n(a, b)$ with $\left(a, b^{n}\right)$ principal. So we assume that $(R, M)$ is local and show that $\operatorname{dim} R=0$ or $R$ is a one-dimensional integral domain. Suppose not. Then there is a nonzero prime ideal $Q \subsetneq M$. Then as in the proof of $(\Leftarrow)$ of Theorem 5, we get a contradiction. Thus for each maximal ideal $M$ of $R$, either $R_{M}$ is zero-dimensional or $R_{M}$ is a one-dimensional domain. Then an application of [K] Exercise 15, page 122], [K, Theorem 167], and [K, Theorem 168] gives that $R$ is a finite direct product of zero-dimensional local rings and one-dimensional domains. (2) $\Rightarrow(1)$ Let $R=R_{1} \times \cdots \times R_{s}$ where each ring $R_{i}$ is either zerodimensional or is a one-dimensional domain. Let $a=\left(a_{1}, \ldots, a_{s}\right), b=\left(b_{1}, \ldots, b_{s}\right) \in$ $R$. Consider $a_{i}, b_{i} \in R_{i}$. Then there exists an $n_{i}$ with $\left(a_{i}, b_{i}^{n_{i}}\right)$ locally principal. Let $n=\max \left\{n_{i}\right\}$. Then $\left(a, b^{n}\right)=\left(a_{1}, b_{1}^{n}\right) \times \cdots \times\left(a_{s}, b_{s}^{n}\right)$ is easily seen to be locally principal.

\section{REFERENCES}

[AMZ] D.D. Anderson, J.L. Mott and M. Zafrullah, Finite character representations for integral domains, Boll. Un. Mat. Ital. B (7) 6 (1992), 613-630. MR 93k:13001

[B] V. Barucci, Mori domains, Non-Noetherian Commutative Ring Theory, Math. Appl., vol. 520, Kluwer Acad. Publ., Dordrecht, 2000, pp. 57-73. MR 2002h:13028

[CMZ] D. Costa, J.L. Mott and M. Zafrullah, The construction $D+X D_{S}[X]$, J. Algebra 53 (1978), 423-439. MR 58:22046

[G] R. Gilmer, Multiplicative Ideal Theory, Queen's Papers in Pure and Appl. Math., vol. 90, Queen's University, Kingston, ON, 1992, corrected reprint of the 1972 edition, Pure Appl. Math., vol. 12, Marcel Dekker, New York. MR 93j:13001

[HH] J.R. Hedstrom and E.G. Houston, Some remarks on star-operations, J. Pure Appl. Algebra 18 (1980), 37-44. MR 81m:13008

[K] I. Kaplansky, Commutative Rings, University of Chicago Press, Chicago, IL, 1974, revised edition of the 1970 edition, Allyn and Bacon, Boston. MR 49:10674

[MMZ] S. Malik, J.L. Mott and M. Zafrullah, On t-invertibility, Comm. Algebra 16 (1988), 149170. MR 88j:13022

[O] J. Ohm, Some counterexamples related to integral closure in $D[[x]]$, Trans. Amer. Math. Soc. 122 (1966), 321-333. MR 34:2613

[P] M. Picavet-L'Hermitte, Factorization in some orders with a PID as integral closure, Algebraic Number Theory and Diophantine Analysis (Graz, 1998), de Gruyter, Berlin, 2000, pp. 365-390. MR 2001h:13001

[R] P. Ribenboim, Anneaux normaux réels à caractère fini, Summa Brasil. Math. 3 (1956), 213-253. MR 20:3860

[Z] M. Zafrullah, Putting t-invertibility to use, Non-Noetherian Commutative Ring Theory, Math. Appl., vol. 520, Kluwer Acad. Publ., Dordrecht, 2000, pp. 429-457. MR 2002g: 13009

Department of Mathematics, University of Iowa, Iowa City, Iowa 52242

E-mail address: dan-anderson@uiowa.edu

Department of Mathematics, Idaho State University, Pocatello, Idaho 83209-8085

E-mail address: mzafrullah@usa.net

URL: http://www.isu.edu/ zafrmuha 